\title{
TSA increases C/EBP- $\alpha$ expression by increasing its lysine acetylation in hepatic stellate cells
}

\author{
LI-LI TAO ${ }^{1}$, DI DING ${ }^{2}$, WEI-HUA YIN ${ }^{1}$, JI-YING PENG ${ }^{1}$, CHEN-JIAN HOU $^{3}$, XIU-PING LIU $^{3,4}$ and YAO-LI CHEN ${ }^{1}$ \\ ${ }^{1}$ Department of Pathology, Peking University, Shenzhen Hospital, Shenzhen, Guangdong 518001; \\ ${ }^{2}$ Department of Pathology, Fudan University Affiliated Zhongshan Hospital; ${ }^{3}$ Department of \\ Pathology, School of Basic Medical Sciences, Fudan University, Shanghai 200032; ${ }^{4}$ Department of \\ Pathology, The Fifth People's Hospital, Fudan University, Shanghai 200040, P.R. China
}

Received October 29, 2016; Accepted July 4, 2017

DOI: $10.3892 / \mathrm{mmr} .2017 .7358$

\begin{abstract}
CCAAT enhancer binding protein- $\alpha$ (C/EBP- $\alpha$ ) is a transcription factor expressed only in certain tissues, including the liver. It has been previously demonstrated that C/EBP- $\alpha$ may induce apoptosis in hepatic stellate cells (HSCs), raising the question of whether acetylation of $\mathrm{C} / \mathrm{EBP}-\alpha$ is associated with HSCs, and the potential associated mechanism. A total of three histone deacetylase inhibitors (HDACIs), including trichostatin A (TSA), suberoylanilide hydroxamic acid and nicotinamide, were selected to determine whether acetylation affects C/EBP- $\alpha$ expression. A Cell Counting Kit- 8 assay was used to determine the rate of proliferation inhibition following treatment with varying doses of the three HDACIs in HSC-T6 and BRL-3A cells. Western blot analysis was used to examine Caspase-3, -8, -9, and -12 levels in HSC-T6 cells treated with adenoviral-C/EBP- $\alpha$ and/or TSA. Following treatment with TSA, a combination of reverse transcription-quantitative polymerase chain reaction and western blot analyses was used to determine the inherent $\mathrm{C} / \mathrm{EBP}-\alpha \mathrm{mRNA}$ and protein levels in HSC-T6 cells at 0, 1, 2, 4, 8, 12, 24, 36 and $48 \mathrm{~h}$. Nuclear and cytoplasmic proteins were extracted to examine $\mathrm{C} / \mathrm{EBP}-\alpha$ distribution. Co-immunoprecipitation analysis was used to examine the lysine acetylation of $\mathrm{C} / \mathrm{EBP}-\alpha$. It was observed that TSA inhibited the proliferation of HSC-T6 cells to a greater extent compared with BRL-3A cells, following treatment with the three HDACIs. TSA induced apoptosis in HSC-T6 cells and enhanced the expression of $\mathrm{C} / \mathrm{EBP}-\alpha$. Following treatment of HSC-T6 cells with TSA, inherent $\mathrm{C} / \mathrm{EBP}-\alpha$ expression increased in a time-dependent manner, and its lysine acetylation simultaneously increased.
\end{abstract}

Correspondence to: Dr Yao-Li Chen, Department of Pathology, Peking University, Shenzhen Hospital, 1120 Lianhua Road, Futian, Shenzhen, Guangdong 518001, P.R. China

E-mail: 09111010055@fudan.edu.cn

Key words: hepatic stellate cells, CCAAT enhancer binding protein- $\alpha$, apoptosis, acetylation
Therefore, the results of the present study suggested that TSA may increase C/EBP- $\alpha$ expression by increasing its lysine acetylation in HSCs.

\section{Introduction}

Liver fibrosis and its end-stage disease (cirrhosis) are major health problems worldwide. Liver fibrosis results in liver failure and portal hypertension, which frequently require liver transplantation at later stages. The activation of hepatic stellate cells (HSCs) serves an important role in the process of liver fibrosis. HSCs are nonparenchymal, quiescent cells that store vitamin A and maintain a normal basement membrane-type matrix in an undamaged liver. However, liver injury induces an 'activation' process in HSCs that results in vitamin A loss, proliferation, and the synthesis of a 'fibrotic' matrix that is rich in type I collagen (1).

CCAAT enhancer binding protein- $\alpha(\mathrm{C} / \mathrm{EBP}-\alpha)$ is a transcription factor expressed only in certain tissues, including the liver $(2,3)$. It was previously demonstrated that C/EBP- $\alpha$ may induce HSC apoptosis in vitro (4) and in vivo (5). In addition, $\mathrm{C} / \mathrm{EBP}-\alpha$ results in differential effects on apoptosis in hepatocytes and HSCs in vitro and in vivo, exerting little hepatocyte toxicity (6). These previous results suggested that C/EBP- $\alpha$ may be an effective anti-liver fibrosis therapy that results in little hepatocyte damage.

Epigenetic mechanisms, including DNA methylation and histone tail modifications, are primary contributors to the function of $\mathrm{C} / \mathrm{EBP}-\alpha$ in numerous types of tumor, including lung cancer (7), acute myeloid leukemia (8), head and neck squamous cell carcinoma (9) and pancreatic cancer (10). However, there are a limited number of studies on the epigenetic modifications of $\mathrm{C} / \mathrm{EBP}-\alpha$ in fibrosis. Therefore, the present study investigated whether the acetylation of C/EBP- $\alpha$ may induce HSC-T6 cellular apoptosis, and the associated mechanism involved. The effects of trichostatin A (TSA), suberoylanilide hydroxamic acid (SAHA) and nicotinamide on the HSC line HSC-T6 and the hepatocyte line BRL-3A were investigated, and it was observed that HSC-T6 cells were more sensitive to TSA compared with BRL-3A cells. Therefore, TSA was selected to further investigate its effect on apoptosis in HSC-T6 cells and the acetylation 
of C/EBP- $\alpha$. The results of the present study will improve understanding of the $\mathrm{C} / \mathrm{EBP}-\alpha$ mechanism in hepatic fibrosis.

\section{Materials and methods}

Materials. Anti-C/EBP- $\alpha$ (cat. no. sc-61-G) was obtained from Santa Cruz Biotechnology, Inc. (Dallas, Texas, USA), horseradish peroxidase (HRP)-conjugated goat anti-mouse immunoglobulin (Ig)G (cat. no. SA0001-1) and goat anti-rabbit $\operatorname{IgG}$ (cat. no. SA0001-1) were obtained from ProteinTech Group, Inc. (Chicago, IL, USA). Anti- $\beta$-actin (A5441) was purchased from Sigma-Aldrich (Merck KGaA, Darmstadt, Germany). Antibodies against Caspase-3 (cat. no. \#9662, which recognizes the procaspase and cleaved Caspase forms), cleaved Caspase-3 (cat. no. \#9661, which detects only the cleaved form), Caspase-8 (cat. no. \#4790, which recognizes the procaspase and cleaved Caspase forms), Caspase- 9 (cat. no. \#9508, which recognizes the procaspase and cleaved Caspase forms), and acetylated lysine (cat. no. \#9441) were purchased from Cell Signaling Technology, Inc. (Danvers, MA, USA). Anti-Caspase-12 (cat. no. 3282-100, which recognizes the procaspase and cleaved Caspase forms) was purchased from BioVision, Inc. (Milpitas, CA, USA). The Cell Counting Kit-8 (CCK-8) assay kit was purchased from Dojindo Molecular Technologies, Inc. (Kumamoto, Japan). NE-PER nuclear and cytoplasmic extraction reagents and the Pierce Co-Immunoprecipitation (co-IP) kit were purchased from Thermo Fisher Scientific, Inc. (Waltham, MA, USA). TRIzol reagent was obtained from Sangon Biotech Co., Ltd. (Shanghai, China). The PrimeScript RT Reagent kit (Perfect Real Time) and SYBR Premix Ex Taq kit (Perfect Real Time) were obtained from Takara Biotechnology Co., Ltd. (Dalian, China). TSA and nicotinamide were purchased from Sigma-Aldrich (Merck KGaA). SAHA was obtained from Cayman Chemical Company (Ann Arbor, MI, USA). Epigallocatechin gallate (EGCG) was obtained from YuanYe Biotechnology Co., Ltd. (Shanghai, China).

Cell culture. The immortalized rat liver stellate cell line HSC-T6 was established by Dr. Scott L. Friedman and generously provided by his student, Professor Lie-Ming Xu (Shanghai University of Traditional Chinese Medicine, Shanghai, China). The rat hepatocyte cell line BRL-3A was purchased from the American Type Culture Collection (Manassas, VA, USA). The two cell lines were cultured in Dulbecco's modified Eagle's medium supplemented with $10 \%$ fetal bovine serum (both from Gibco; Thermo Fisher Scientific, Inc.) and standard antibiotics in a $95 \%$ air and $5 \% \mathrm{CO}_{2}$ humidified atmosphere at $37^{\circ} \mathrm{C}$.

Construction of the C/EBP- $\alpha$ replication-defective recombinant adenovirus (Ad-C/EBP- $\alpha$ ). The plasmid PDC316 was constructed in the lab previously (4). Adenovirus construction, amplification, purification and titer detection were performed as described previously (4).

CCK- 8 assay. HSC-T6 and BRL-3A cells were seeded onto 96-well plates at a density of $1 \times 10^{5}$ cells/well. Cells were treated with varying concentrations of TSA, SAHA and nicotinamide for $48 \mathrm{~h}$ at $37^{\circ} \mathrm{C}$ (Fig. 1). Cell viability was measured using the CCK-8 kit. Control cells were treated with $10 \%$ dimethyl sulfoxide for $48 \mathrm{~h}$. Following the addition of the test compounds, $10 \mu \mathrm{l}$ CCK- 8 was added to each well, incubated for $1-3 \mathrm{~h}$ at $37^{\circ} \mathrm{C}$, and optical density was read at a wavelength of $450 \mathrm{~nm}$ in a VMax kinetic microplate reader (Molecular Devices, LLC, Sunnyvale, CA, USA).

Reverse transcription-quantitative polymerase chain reaction (RT-qPCR) analysis. Following treatment with TSA for the indicated times $(0,1,2,4,8,12,24,36$ and $48 \mathrm{~h})$ at concentration of $0.1 \mu \mathrm{mol} / 1$, total RNA from HSC-T6 cells was extracted using TRIzol reagent, according to the manufacturer's protocol. Briefly, total RNA (1 $\mu \mathrm{g})$ was reverse-transcribed using a PrimeScript RT kit (Takara Biotechnology Co., Ltd.) with oligodT primer and six random primers, to obtain the cDNA. The cDNA was amplified by PCR. PCR was performed in a Rotor Gene thermal cycler (RG-3000; Qiagen GmbH, Hilden, Germany) under conditions optimized for efficient amplification of the respective genes and the reference gene. Amplification was performed as follows: Following the initial activation step at $95^{\circ} \mathrm{C}$ for $5 \mathrm{~min}, 40$ cycles of denaturation at $95^{\circ} \mathrm{C}$ for $5 \mathrm{sec}$ and annealing at $60^{\circ} \mathrm{C}$ for $30 \mathrm{sec}$ were performed. qPCR (11) experiments were conducted in three biological replicates. The following primer sequences were used: Rat C/EBP- $\alpha, 5^{\prime}$ CGGTGGATAAGAACAGCAACGA3' (sense) and 5'GCGGTCATTGTCACTGGTCAAC3' (antisense); and rat $\beta$-actin, 5'AGGATGCAGAAGGAGATTACTGC3' (sense) and 5'AAAACGCAGCTCAGTAACAGTGC 3' (antisense).

Western blot analysis. Following treatment at $37^{\circ} \mathrm{C}$ with $0.1 \mu \mathrm{mol} / 1 \mathrm{TSA}$ for the indicated times $(0,1,2,4,8,12,24,36$ and $48 \mathrm{~h}$ ), or treatment with TSA and/or Ad-C/EBP- $\alpha$ with a range of dosages, HSC-T6 cells were collected, washed in cold PBS, and subsequently lysed in ice-cold radioimmunoprecipitation assay buffer $[10 \mu \mathrm{M}$ Tris- $\mathrm{HCl}(\mathrm{pH} 8.0), 100 \mathrm{mM}$ $\mathrm{NaCl}, 1 \mathrm{mM}$ EDTA, $1 \%$ Nonidet P-40, 0.5\% sodium deoxycholate, $0.1 \%$ SDS, and $10 \mu \mathrm{l} / \mathrm{ml}$ protease inhibitor cocktail] on ice for $45 \mathrm{~min}$. Lysates were cleared by centrifugation at $13,000 \mathrm{x} \mathrm{g}$ for $30 \mathrm{~min}$ at $4^{\circ} \mathrm{C}$, and total protein concentration was determined using a Bicinchoninic Acid Protein Assay kit. Extracted cellular proteins $(10 \mu \mathrm{g})$ from each sample were denatured in SDS containing sample buffer, applied to a 10-12\% SDS-PAGE gel and transferred to nitrocellulose membranes. Following blocking in $5 \%$ non-fat milk for $1 \mathrm{~h}$ at room temperature, the membranes were incubated overnight at $4^{\circ} \mathrm{C}$ with primary antibodies against anti-C/EBP- $\alpha$ (1:200), anti-Caspase-3 (1:1,000), anti-Caspase-8 $(1: 1,000)$, anti-Caspase-9 $(1: 1,000)$ or anti-Caspase-12 $(1: 1,000)$, respectively. $\beta$-actin $(1: 1,000)$ was used as a loading control. Membranes were subsequently incubated for $1 \mathrm{~h}$ at $4^{\circ} \mathrm{C}$ with the appropriate HRP-conjugated secondary antibody. Protein bands were visualized using an Enhanced Chemiluminescence Assay kit (Thermo Fisher Scientific, Inc.). ImageJ 1.50e (National Institutes of Health, Bethesda, MD, USA) was used for densitometric analysis.

Nuclear and cytoplasmic protein extraction. HSC-T6 cells $\left(\sim 1 \times 10^{6}\right)$ were harvested following treatment with $0.1 \mu \mathrm{mol} / 1$ TSA for $12 \mathrm{~h}$ at $37^{\circ} \mathrm{C}$. Cells were washed in cold PBS, and nuclear and cytoplasmic proteins were extracted using NE-PER 
Effects on BRL-3A and HSC-T6 induced by different HDACls

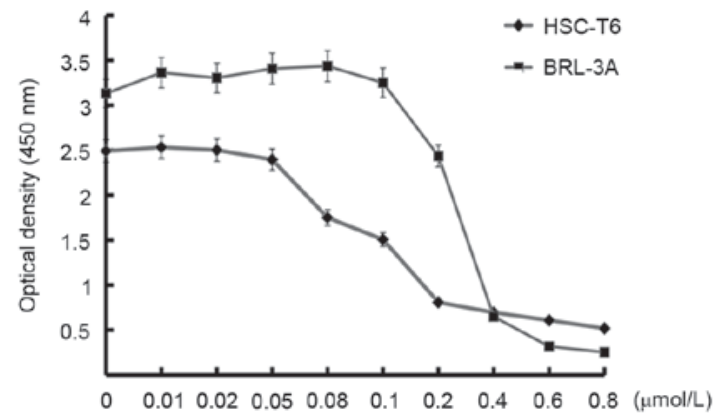

TSA, $48 \mathrm{~h}$

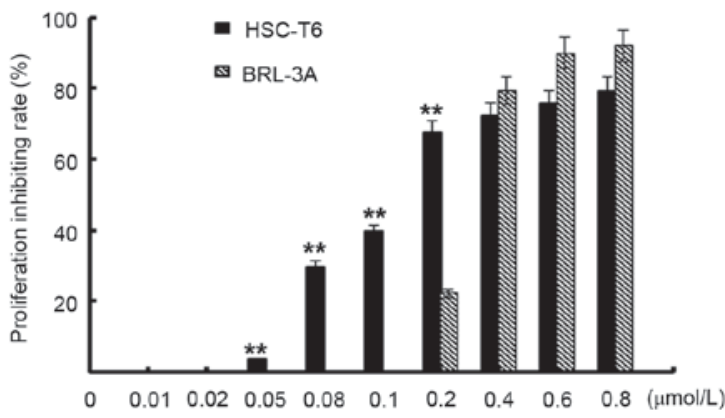

D

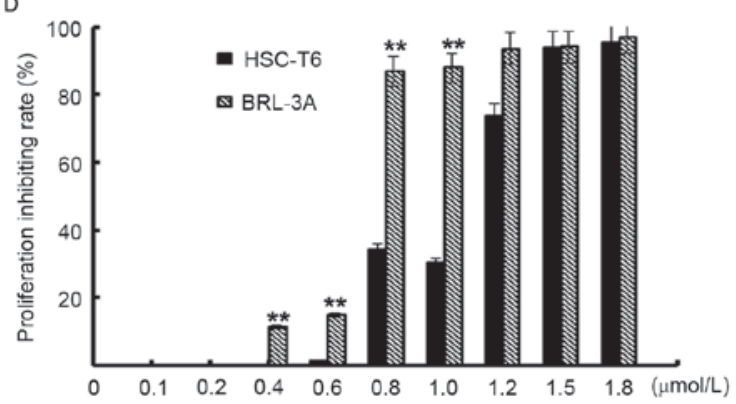

C

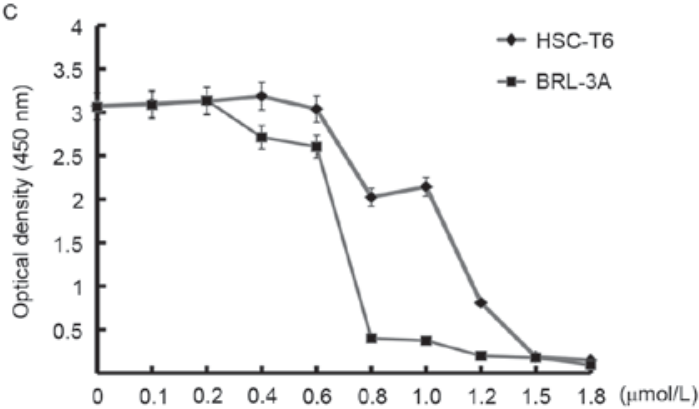

SAHA, $48 \mathrm{~h}$
$\mathrm{E}$

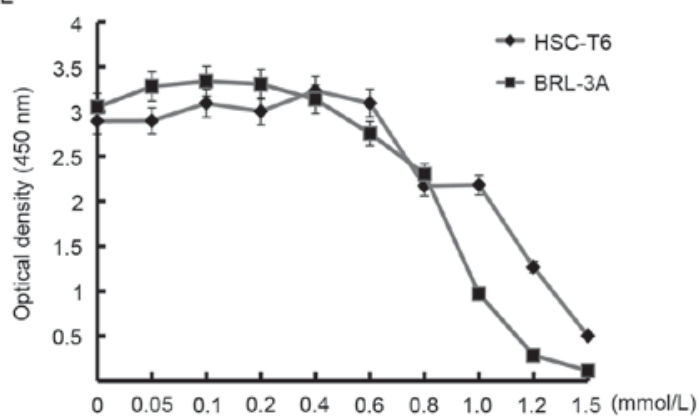

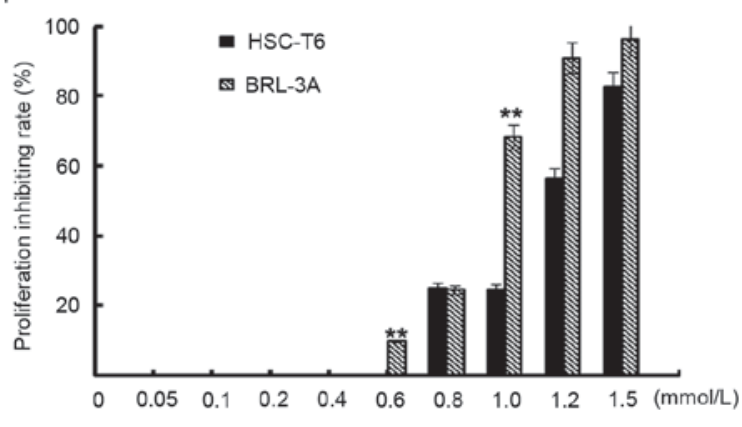

Nicotinamide, $48 \mathrm{~h}$

Figure 1. Inhibitory effects of TSA, SAHA and nicotinamide on BRL-3A and HSC-T6 cells. (A) Optical density and (B) growth inhibition rate of BRL-3A and HSC-T6 cells following treatment with varying doses of TSA. (C) Optical density and (D) growth inhibition rate of BRL-3A and HSC-T6 cells following treatment with varying doses of SAHA. (E) Optical density and (F) growth inhibition rate of BRL-3A and HSC-T6 cells following treatment with varying doses of nicotinamide. ${ }^{* *} \mathrm{P}<0.01$ vs. BRL-3A. TSA, trichostatin A; SAHA, suberoylanilide hydroxamic acid; HDACIs, histone deacetylase inhibitors.

nuclear and cytoplasmic extraction reagents, according to the manufacturer's protocol. Proteins were stored at $-80^{\circ} \mathrm{C}$ until ready for use in subsequent experiments.

Co-IP analysis of $C / E B P-\alpha$ and the acetylated lysine antibody in HSC-T6 cells. Following treatment with TSA at a concentration of $0.1 \mu \mathrm{mol} / 1$ for $12 \mathrm{~h}$ at $37^{\circ} \mathrm{C}$, co-IP analysis of C/EBP- $\alpha$ and the acetylated lysine antibody was performed in HSC-T6 cells using a co-IP kit, according to the manufacturer's protocol. C/EBP- $\alpha$ and the acetylated lysine antibody were coupled to AminoLink Plus Coupling Resin. HSC-T6 cell lysate was prepared with IP lysis/wash buffer on ice for $5 \mathrm{~min}$. Purified HSC-T6 cell lysate was incubated with $\mathrm{C} / \mathrm{EBP}-\alpha$, acetylated lysine antibody-specific $\mathrm{MAb}$-conjugated agarose resin overnight at $4^{\circ} \mathrm{C}$. Following elution, proteins samples were prepared for western blot analysis using antibodies against $\mathrm{C} / \mathrm{EBP}-\alpha$ or acetylated

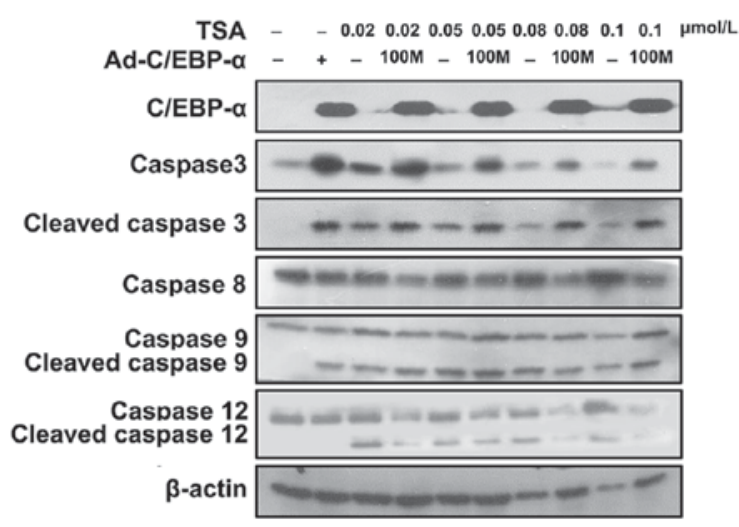

Figure 2. Expression of Caspase-3, -8, -9, and -12 in different groups of HSC-T6 cells determined by western blot analysis. Cells were treated with varying doses of TSA and/or 100 multiplicity of infection Ad-C/EBP- $\alpha$. Ad, adenoviral; C/EBP- $\alpha$, CCAAT enhancer binding protein- $\alpha$; TSA, trichostatin, A. 
A

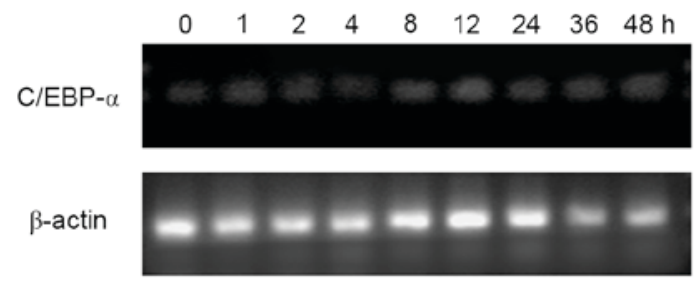

C

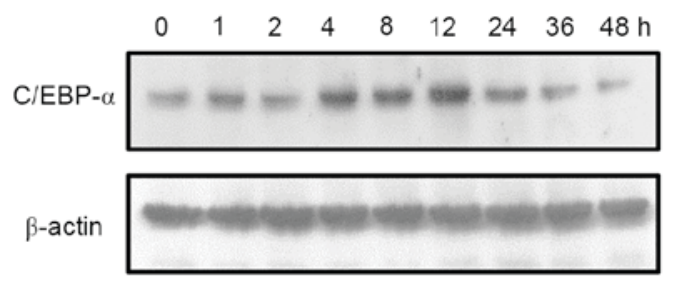

B

Relative expression of C/EBP- $\alpha$ mRNA to $\beta$-actin

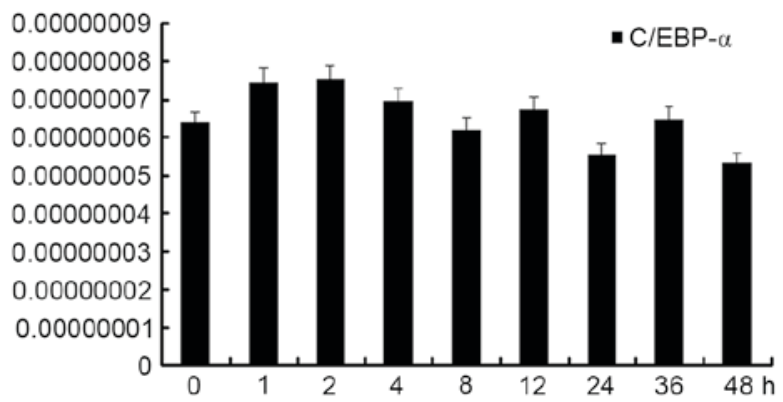

D

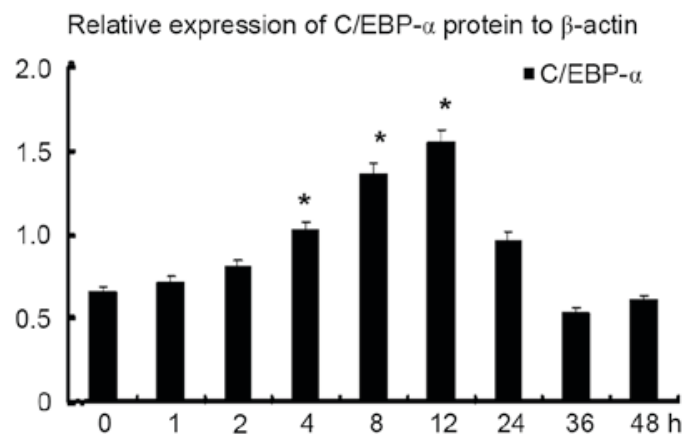

Figure 3. C/EBP- $\alpha$ mRNA and protein levels following treatment with TSA at the indicated times. (A) Representative image of C/EBP- $\alpha$ mRNA level following treatment with TSA for $0,1,2,4,8,12,24,36$ and $48 \mathrm{~h}$, demonstrated by agarose gel electrophoresis. (B) C/EBP- $\alpha$ mRNA level following treatment with TSA at $0,1,2,4,8,12,24,36$ and $48 \mathrm{~h}$ as determined by quantitative polymerase chain reaction analysis. (C) C/EBP- $\alpha$ protein level following treatment with TSA at $0,1,2,4,8,12,24,36$ and $48 \mathrm{~h}$, analyzed by western blotting. (D) Densitometric analysis of the protein expression of C/EBP- $\alpha$. "P<0.05 vs. control. C/EBP- $\alpha$, CCAAT enhancer binding protein- $\alpha$; TSA, trichostatin A.

lysine as aforementioned. A sample of the whole input lysate served as a positive control, and normal mouse IgG (Beyotime Institute of Biotechnology, Haimen, China) was used as negative control.

Statistical analysis. All experiments were repeated three independent times. Statistical analysis was performed using SPSS software, version 11.0 (SPSS, Inc., Chicago, IL, USA). Data were expressed as the mean \pm standard deviation. Comparisons were performed using Student's t-test. For multiple comparisons, repeated measures analysis of variance and Dunnett's test were used. $\mathrm{P}<0.05$ was considered to indicate a statistically significant difference.

\section{Results}

TSA inhibits the proliferation of HSC-T6 cells to a greater extent compared with the proliferation of BRL-3A cells, following treatment with the three histone deacetylase inhibitors (HDACIs). HSC-T6 and BRL-3A cells exhibited differential responses to the three HDACIs. HSC-T6 cells were more sensitive to TSA compared with BRL-3A cells. Proliferation was inhibited by $0.05 \mu \mathrm{mol} / 1$ and $0.2 \mu \mathrm{mol} / 1$ TSA. The rate of proliferation inhibition was $67.54 \%$ in HSC-T6 cells and 22.21\% in BRL-3A cells $(\mathrm{P}<0.01)$. However, compared with treatment with TSA, BRL-3A cells were more sensitive to SAHA and nicotinamide (Fig. 1). The ideal medicine to treat liver fibrosis is one that is able to inhibit HSCs while exerting a small effect on hepatocytes. Therefore, TSA was selected for the subsequent experiments in HSC-T6 cells.
Treatment with TSA alone or in combination with Ad-C/EBP- $\alpha$ induces the expression of apoptotic markers in HSC-T6 cells. Varying doses of TSA alone or in combination with 100 multiplicity of infection (MOI) Ad-C/EBP- $\alpha$ were used to detect the expression of apoptotic markers of Caspase-3, -8, -9 and -12 levels in HSC-T6 cells (Fig. 2). It was observed that TSA alone and in combination with $100 \mathrm{MOI}$ Ad-C/EBP- induced apoptosis in HSC-T6 cells. Treatment with TSA induced apoptosis through Caspase-9 and Caspase-12, whereas Ad-C/EBP- $\alpha$ induced apoptosis primarily through the Caspase- 9 pathway. However, activated Caspase- 8 was not detected. These results suggested that TSA may enhance C/EBP- $\alpha$-induced apoptosis of HSCs.

Inherent C/EBP-a protein expression in HSC-T6 cells varies, although $m R N A$ does not alter. In order to further investigate the effect of TSA on C/EBP- $\alpha$ expression in HSC-T6 cells, they were first treated with TSA at concentration of $0.1 \mu \mathrm{mol} / 1$, and subsequently harvested for RT-qPCR and western blot analyses at $0,1,2,4,8,12,24,36$ and $48 \mathrm{~h}$. RT-qPCR analysis demonstrated that $\mathrm{C} / \mathrm{EBP}-\alpha$ mRNA expression did not alter significantly, however it was observed that $\mathrm{C} / \mathrm{EBP}-\alpha$ protein expression increased and reached a peak between 4 and $12 \mathrm{~h}$, prior to decreasing gradually, indicating that $\mathrm{C} / \mathrm{EBP}-\alpha$ may be processed post-transcriptionally (Fig. 3).

Inherent $C / E B P-\alpha$ protein expression increases primarily in the nuclei of HSC-T6 cells. Following treatment with TSA for $12 \mathrm{~h}$ at a concentration of $0.1 \mu \mathrm{mol} / 1$, cells were harvested to examine the nuclear and cytoplasmic proteins. C/EBP- $\alpha$ 
A

Cytoplasmic protein

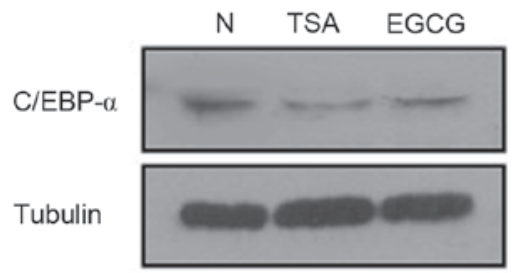

C

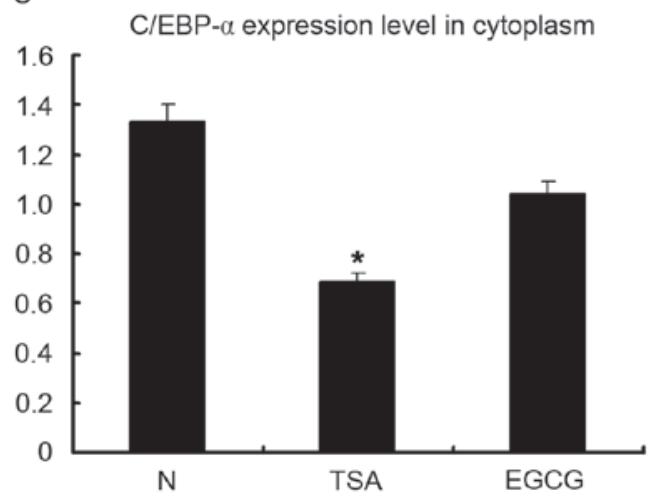

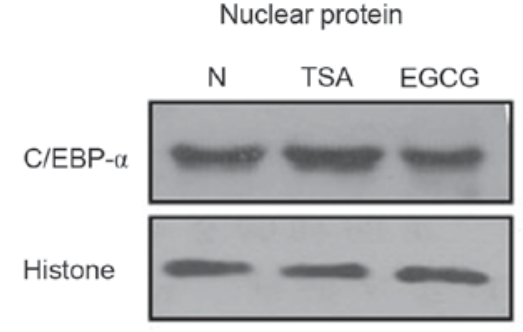

$\mathrm{D}$

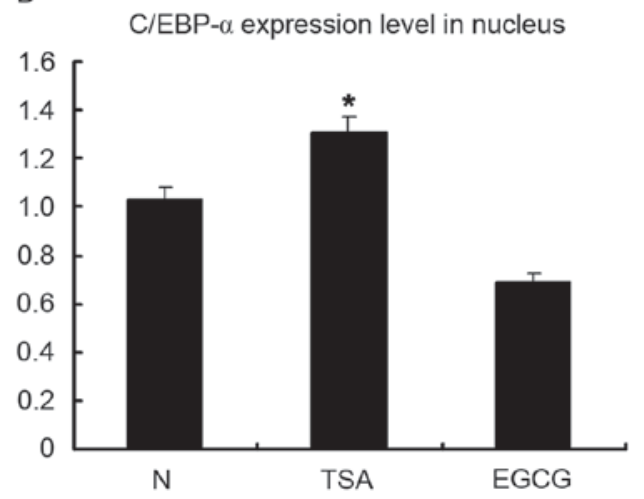

Figure 4. Inherent C/EBP- $\alpha$ protein in nuclear and cytoplasmic fractions, as determined by Western blot analysis. (A) C/EBP- $\alpha$ protein expression in the cytoplasmic fraction following treatment with TSA. (B) C/EBP- $\alpha$ protein expression in the nuclear fraction following treatment with TSA. (C) Densitometric analysis of $\mathrm{C} / \mathrm{EBP}-\alpha$ protein expression in the cytoplasmic fraction. (D) Densitometric analysis of C/EBP- $\alpha$ protein expression in the nuclear fraction. ${ }^{*} \mathrm{P}<0.05$ vs. N group. C/EBP- $\alpha$, CCAAT enhancer binding protein- $\alpha$; TSA, trichostatin A; EGCG, epigallocatechin gallate; N, negative control.

A

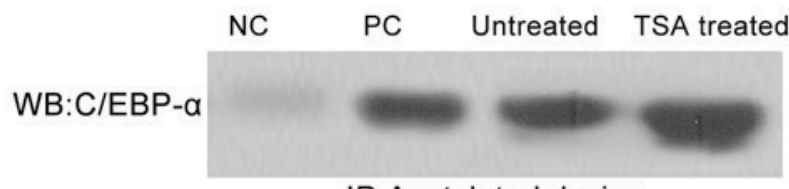

IP:Acetylated lysine
B

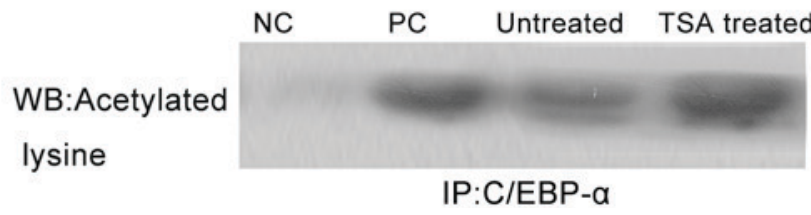

$\mathrm{IP}: \mathrm{C} / \mathrm{EBP}-\alpha$

Figure 5. C/EBP- $\alpha$ lysine acetylation, as determined by co-immunoprecipitation analysis in HSC-T6 cells. (A) Cell lysates were subjected to immunoprecipitation with the C/EBP- $\alpha$ antibody, followed by immunoblotting with anti-acetylated lysine, following treatment with TSA. (B) Cell lysates were subjected to immunoprecipitation with the acetylated lysine antibody, followed by immunoblotting with $\mathrm{C} / \mathrm{EBP}-\alpha$, following treatment with TSA. C/EBP- $\alpha$, CCAAT enhancer binding protein- $\alpha$; TSA, trichostatin A; WB, western blotting; NC, negative control; PC, positive control; IP, immunoprecipitation.

protein expression was decreased in the cytoplasm following treatment with EGCG, which served as the negative control (Fig. 4). However, it was observed that C/EBP- $\alpha$ protein expression was increased in the nuclear fraction following treatment with TSA, verifying that $\mathrm{C} / \mathrm{EBP}-\alpha$ predominantly functions in the nucleus.

C/EBP- $\alpha$ lysine acetylation increases following treatment with TSA, as determined by co-IP analysis. Following treatment with TSA for $12 \mathrm{~h}$ at concentration of $0.1 \mu \mathrm{mol} / 1$, cells were harvested to examine the lysine acetylation of C/EBP- $\alpha$ by co-IP analysis. As presented in Fig. 5, immunoprecipitation of cell lysates with the anti-acetylated lysine antibody coprecipitated with the $\mathrm{C} / \mathrm{EBP}-\alpha$ antibody, and the $\mathrm{C} / \mathrm{EBP}-\alpha$ antibody additionally coprecipitated with the anti-acetylated lysine antibody. Western blot analysis was performed to detect the expected bands; compared with untreated cells, lysine acetylation increased following treatment with TSA.

\section{Discussion}

The exponential growth in the amount of research into HDACs has been driven by the ability of HDACIs to modulate transcriptional activity. HDACIs are able to block angiogenesis and the cell cycle, in addition to promoting apoptosis and differentiation (12. They belong to a heterogeneous class of compounds that includes derivatives of short chain fatty acids, hydroxamic acids, cyclic tetrapeptides, and benzamides. Of the hydroxamic acids, TSA and SAHA are commonly used HDACIs. It has been reported that TSA exerts a potent antifibrogenic effect on HSCs (13). It is possible that TSA induces or maintains the expression of genes essential for the quiescent phenotype of stellate cells. Alternatively, gene products induced by TSA may interfere with the signaling pathways involved in stellate cell activation. Thirdly, TSA may directly repress the transcription of genes that are induced during the activation process (14). In the present study, three HDACIs were applied to determine 
their inhibitory effects on the growth of HSC-T6 and BRL-3A cells. The results demonstrated that HSC-T6 cells were more sensitive to TSA compared with BRL-3A cells; therefore, TSA was selected for use in the subsequent experiments.

The mechanisms underlying apoptosis are complex and involve an energy-dependent cascade of molecular events. Protein expression of Caspase-3, $-8,-9$, and -12 was evaluated in the present study, in order to investigate the apoptotic pathways in HSC-T6 and BRL-3A cells. A previous study demonstrated that Caspase-9 was activated in Ad-C/EBP- $\alpha$-induced apoptosis (6). In the present study, it was observed that TSA induced HSC-T6 cellular apoptosis through the Caspase-9 and -12 pathways. However, the Caspase-12 pathway may be inhibited by co-action with Ad-C/EBP- $\alpha$.

Post-translational modifications (PTMs) are important for regulating the functions of a number of eukaryotic proteins. The lysine side chain is thus a target of numerous PTMs. Lysine acetylation is now known to occur in $>80$ transcription factors, multiple nuclear regulators and various cytoplasmic proteins (14). Lysine residues are targets for acetylation and ubiquitination, and one of these modifications appears to prevent the other. Deacetylation by HDACs, in numerous cases, is a prerequisite for subsequent ubiquitination. Conversely, acetylation may protect a protein from ubiquitination and degradation (15). Whether C/EBP- $\alpha$ ubiquitination is involved requires further investigation.

In conclusion, the results of the present study suggested that TSA may increase C/EBP- $\alpha$ expression by increasing its lysine acetylation in HSCs. However, the probable mechanism requires further investigation. There are few reports on $\mathrm{C} / \mathrm{EBP}-\alpha$ and its acetylation in hepatic fibrosis. However, it is essential to understand this mechanism in order to treat hepatic fibrosis.

\section{Acknowledgements}

The present study was supported by the PhD Start-up Fund of the Natural Science Foundation of Guangdong Province (grant no. 2015A030310031), the Health and Family Planning System of Scientific Research Project of Shen Zhen (grant no. 201401048), Perking University, Shenzhen Hospital Science and Research Foundation (grant no. 201401), the National Natural Science Foundation of China (grant no. 81470857, to X.P.L.), and the Shanghai Natural Science Foundation (grant no. $134119 b 1100)$.

\section{References}

1. Reeves HL and Friedman SL: Activation of hepatic stellate cells-A key issue in liver fibrosis. Front Biosci 7: d808-d826, 2002.

2. Mueller BU and Pabst T: C/EBP alpha and the pathophysiology of acute myeloid leukemia. Curr Opin Hematol 13: 7-14, 2006.

3. Lane MD, Tang QQ and Jiang MS: Role of the CCAAT enhancer binding proteins (C/EBPs) in adipocyte differentiation. Biochem Biophys Res Commun 266: 677-683, 1999.

4. Wang X, Huang G, Mei S, Qian J, Ji J and Zhang J: Over-expression of C/EBP-alpha induces apoptosis in cultured rat hepatic stellate cells depending on p53 and peroxisome proliferator-activated receptor-gamma. Biochem Biophys Res Commun 380: 286-291, 2009.

5. Mei S, Wang X, Zhang J, Qian J and Ji JL: In vivo transfection of C/EBP-alpha gene could ameliorate CCL(4)-induced hepatic fibrosis in mice. Hepatol Res 37: 531-539, 2007.

6. Tao LL, Cheng YY, Ding D, Mei S, Xu JW, Yu J, Ou-Yang Q, Deng $\mathrm{L}$, Chen $\mathrm{Q}$, Li QQ, et al: C/EBP- $\alpha$ ameliorates $\mathrm{CCl}(4)$-induced liver fibrosis in mice through promoting apoptosis of hepatic stellate cells with little apoptotic effect on hepatocytes in vitro and in vivo. Apoptosis 17: 492-502, 2012.

7. Tada Y, Brena RM, Hackanson B, Morrison C, Otterson GA and Plass C. Epigenetic modulation of tumor suppressor CCAAT/Enhancer binding protein a activity in lung cancer. J Natl Cancer Inst 98: 396-406, 2006.

8. Hackanson B, Bennett KL, Brena RM, Jiang J, Claus R, Chen SS Blagitko-Dorfs N, Maharry K, Whitman SP, Schmittgen TD, et al: Epigenetic modification of CCAAT/enhancer binding protein alpha expression in acute myeloid leukemia. Cancer Res 68: 3142-3151, 2008

9. Bennett KL, Hackanson B, Smith LT, Morrison CD, Lang JC, Schuller DE, Weber F, Eng C and Plass C: Tumor suppressor activity of CCAAT/enhancer binding protein alpha is epigenetically down-regulated in head and neck squamous cell carcinoma. Cancer Res 67: 4657-4664, 2007

10. Kumagai T, Akagi T, Desmond JC, Kawamata N, Gery S, Imai Y, Song JH, Gui D, Said J and Koeffler HP: Epigenetic regulation and molecular characterization of C/EBP alpha in pancreatic cancer cells. Int J Cancer 124: 827-833, 2009.

11. Livak KJ and Schmittgen TD: Analysis of relative gene expression data using real-time quantitative PCR and the 2(-Delta Delta C(T)) method. Methods 25: 402-408, 2001.

12. Gridelli C, Rossi A and Maione P: The potential role of histone deacetylase inhibitors in the treatment of non-small-cell lung cancer. Crit Rev Oncol Hematol 68: 29-36, 2008.

13. Niki T, Rombouts K, De Bleser P, De Smet K, Rogiers V, Schuppan D, Yoshida M, Gabbiani G and Geerts A: A histone deacetylase inhibitor, trichostatin A, suppresses myofibroblastic differentiation of rat hepatic stellate cells in primary culture. Hepatology 29: 858-867, 1999.

14. Yang XJ and Seto E: Lysine acetylation: Codified crosstalk with other posttranslational modifications. Mol Cell 31: 449-461, 2008.

15. Glozak MA, Sengupta N, Zhang X and Seto E: Acetylation and deacetylation of non-histone proteins. Gene 363: 15-23, 2005. 